\title{
Influence du vieillessement sur la spermatogenèse : étude histologique et cytogénétique moléculaire au niveau testiculaire chez 46 sujets âgés de 29 à 102 ans
}

\author{
Mbarka DAKOUANE1, Lucia BICCHIERAY1, Marianne BERGERE1,3,4, Martine ALBERT1,3,4 \\ Nathalie LEDEE $^{3}$, François VIALARD ${ }^{3,4}$, Olivier CUSSENOT ${ }^{2}$, Jacqueline SELVA $1,3,4$ \\ 1 Laboratoire d'Histologie Embryologie, UFR des Saint Pères,Université Paris V, Paris ; \\ 2 Laboratoire CEREPP, Paris ; \\ $3 \mathrm{CHI}$ Poissy Service Histo-Embryologie-Biologie de la Reproduction-Cytogénétique, Poissy ; \\ 4 INSERM Unité 407, Oulins
}

\section{RESUME}

L'effet délétère de l'âge maternel sur le risque d'anomalie méiotique est bien établi, mais il n'en est pas de même pour l'effet de l'âge paternel. Nous avons étudié l'influence du vieillissement sur la spermatogenèse évaluée par l'histologie testiculaire évaluée par histomorphomètrie et sur le taux d'anomalies méiotiques testiculaires évalué par la technique d'hybridation in situ fluorescente (FISH) pour les chromosomes $X, Y$ et 18. L'étude a comporté 46 hommes âgés de 29 à 102 ans (36 sujets âgés et 10 témoins). L'analyse histomorphomètrique des biopsies testiculaires a concerné les tubes séminifères : surface, épaisseur de la membrane propre, densité en cellules de Sertoli, en spermatogonies, en spermatocytes et en spermatozoïdes, ainsi que le tissu interstitiel : nombre de groupements des cellules de Leydig par champ, surface occupée par les cellules de Leydig, et densité. Cette étude nous a permis de classer les sujets âgés $(n=36)$ en trois groupes: 17 sujets âgés de 61 à 95 ans à spermatogenèse complète (groupe 1), 4 sujets à spermiogenèse arrêtée (groupe 2) et 15 sujets avec un arrêt précoce de la spermatogenèse (cellules diploïdes) (groupe 3 ). Les variations interindividuelles étaient très importantes. L'épaisseur de la membrane propre paraît être le paramètre le mieux corrélé à la conservation de la spermatogenèse. Nous avons analysé par FISH un total de 10000 cellules postméiotiques dont 4738 cellules post méiotiques dans le groupe 1 et un total de 4882 cellules post méiotiques chez les sujets jeunes témoins. Les taux des cellu. les normales haploïdes $X 18$ et $Y 18$ représentent respectivement $50,76 \%$ et $47,95 \%$ dans le groupe de sujets âgés (groupe 1), $50,35 \%$ et $48,55 \%$ dans le groupe des témoins. Le taux d'aneuploïdie totale est de 1,29\% chez les sujets âgés du groupe 1 et de $1,10 \%$ chez le groupe témoin. Pour le groupe 2 (sujets avec un arrêt de la spermiogenèse), les pourcentages de cellules X18, Y18 et le taux d'aneuploïdie étaient respectivement $47,96 \%, 37,75 \%$ et $14,28 \%$ au niveau des spermatides ( $n=98$ ). Ce taux d'anomalies de $14,28 \%$ est significativement accru, comparé aux résultats du groupe 1 et des témoins.

Au total nous avons conclu qu'une spermatogenèse peut être conservée à un âge très avancé (95 ans), que le taux d'aneuploïdie postméiotique des sujets âgés à spermatogenèse complète n'est pas globalement différent de celui qui est observé chez des sujets jeunes témoins et que l'incidence des aneuploïdies post-méiotiques semble être plus élevée lorsque la spermiogenèse est arrêtée $(14,28 \%$ versus $1,10 \%$ ).

Mots clés : vieillissement, testicules, histomorphomètrie, spermatozoïdes, FISH et anomalie chromosomique

\section{Correspondance:}

Dr Mbarka DAKOUANE - Laboratoire d'Histologie Embryologie, UFR des Saint Pères, Université Paris V, 45 rue des Saints Pères, 75270 Paris Cedex 06 - Tel 01.39.27.47.09 - Email dakouane@hotmail.com 


\section{INTRODUCTION}

Les hommes produisent de manière continue des spermatozoïdes et ceci durant toute la vie (à partir de la puberté). Le nombre de divisions des cellules germinales préalables à la production de spermatozoïdes augmente avec l'âge. Or on peut penser que cette augmentation du nombre de divisions avec l'âge puisse induire des accidents génétiques au niveau germinal. Chez la femme la production d'ovocytes diminue dès l'âge de 30 à 40 ans [17]. Autour des 50 ans, la fertilité féminine s'arrête totalement (ménopause). A cet âge, on estime que le nombre de divisions cellulaires préalables à la production d'un ovocyte est de 24 tandis que chez un homme âgé de 45 ans le nombre de divisions cellulaires préalables à la production des spermatozoïdes est d'environ 770 [6]. Ainsi, un homme peut être père à un âge avancé mais avec un risque probablement accru de mutation génétique.

Plusieurs études ont montré qu'avec l'âge, le volume spermatique et la mobilité des spermatozoïdes diminuent, de même que la morphologie des spermatozoïdes se modifie $[7,15]$. Par ailleurs, les investigations histologiques effectuées sur des testicules prélevés sur des hommes âgés ont rapporté l'existence d'une artériosclérose systémique [30, 35] et d'une involution des cellules de Leydig [29]. Une corrélation positive entre la proportion de cellules de Leydig altérées et la diminution du niveau de testostérone a également èté rapportée $[23,27]$. De plus chez les hommes âgès, l'épaisseur de la membrane basale des tubes séminifères augmente [20]. On observe également des changements dans la structure des cellules de Sertoli [26]. Enfin, dans les testicules de sujets âgés il a aussi été observé une dégénérescence des cellules germinales ainsi qu'une diminution progressive des spermatozoïdes ou un arrêt complet au stade pachytène $[11,12,25,5]$.

Le risque d'accident génétique lié à l'âge paternel est une question discutée et d'actualité du fait d'une fréquence accrue de demandes d'assistance médicale à la procréation (AMP) par des couples dont le conjoint est âgé. Si l'effet délétère de l'âge maternel sur le risque d'anomalie méiotique est bien établi [28], en revanche, nous ne disposons que de peu de données concernant l'effet de l'âge paternel sur l'incidence des anomalies chromosomiques au cours de la méiose. Les résultats des études traitant cette question sont plutôt controversés et le nombre de sujets étudiés âgés de plus 60 ans est trop faible pour tirer des conclusions définitives.

Ces divergences nous ont conduit à étudier l'influence du vieillissement sur la spermatogenèse évaluée par l'histologie testiculaire et sur le taux d'anomalies méiotiques testiculaires évalué par la technique d'hybridation in situ fluorescente (FISH) pour les chromosomes $X, Y$ et 18, effectuée sur les cellules germinales obtenues par empreinte testiculaire. Nous avons choisi d'étudier les chromosomes $X$ et $Y$ pour plusieurs raisons : d'une part les anomalies des chromosomes sexuels observées à la naissance sont souvent d'origine paternelle $[10,24]$; d'autre part les malségrégations $X Y$ sont plus fréquentes dans le sperme des patients oligospermes que chez les contrôles $[1,34]$; enfin les chromosomes sexuels permettent de voir à quelle étape la méiose est arrêtée (méiose 1 ou 2 ) ou bien à quelle étape la non disjonction est intervenue. Le chromosome 18 nous a permis de vérifier la ploïdie des cellules examinées ainsi que l'incidence des malségrégations pour le chromosome 18 dont les trisomies sont viables.

\section{MATERIEL ET METHODES}

\section{Sujets}

II s'agit de 35 hommes décédés entre l'âge de 65 et 102 ans et ayant donné leur corps au Centre du Don du Corps des Saints-Pères (Université Paris V). Les testicules ont été prélevés dans un délai n'excédant pas les 36 heures post mortem. Cette étude inclut également un patient âgé de 61 ans consultant pour AMP suite à une vasectomie et qui avait été fertile avant sa vasectomie. Les 10 témoins sont des sujets jeunes âgés de 26 à 40 ans présentant une azoospermie obstructive avec une histologie testiculaire normale.

\section{Histologie}

Pour chaque sujet, trois fragments ont été prélevés sur chaque testicule. Ces fragments ont été fixés dans le Bouin et inclus dans la paraffine. Des coupes minces $(7 \mu \mathrm{m})$ ont été colorées au trichrome de Masson. Pour chaque coupe histologique, six tubes séminifères ont été analysés au grossissement $X 400$. L'étude histomorphomètrique des biopsies testiculaires a été réalisée à l'aide du logiciel Histolab (version 5.2.3 ; Microvision Instrument, France). Concernant les tubes séminifères, nous avons mesuré leur surface, l'épaisseur de la membrane propre, la densité en cellules de Sertoli, en spermatogonies, en spermatocytes et en spermatozoïdes. Concernant le tissu interstitiel, nous avons évalué le nombre de groupements de cellules de Leydig par champ, la surface occupée par les cellules de Leydig et leur densité.

\section{Hybridation in situ fluorescente (FISH)}

\section{a) Préparation des empreintes testiculaires.}

Les empreintes testiculaires ont été réalisées sur des lames propres (Super frost plus slides) et ont été stockées au congélateur à $-20^{\circ} \mathrm{C}$. Au total, 8 lames ont été préparées pour chaque sujet. Pour les témoins, des frottis ont été réalisés à partir des fragments de biopsie testiculaire dilacérés.

\section{b) Préparation des lames.}

Les lames ont été fixées par un mélange méthanol acide acétique pendant 5 minutes. Elles ont ensuite été immergées dans du $2 X S S C(0,3 \mathrm{M} \mathrm{NaCl}, 0,03 \mathrm{M}$ trisodium citrate $2 \mathrm{H} 2 \mathrm{O}, \mathrm{pH} 7,0$ ) à température ambiante pendant 5 à 10 minutes, puis soumises à un lavage au PBS durant 5 minutes. Les lames ont ensuite été déshydratées par immersion dans des bains d'alcool de degrés croissants $\left(80^{\circ}, 90^{\circ}\right.$ et $100^{\circ}$ ) pendant 1 à 2 minutes dans chaque bain, puis séchées à température ambiante. 


\section{c) Choix de la zone à hybrider.}

La localisation de la zone à hybrider a été choisie au microscope à contraste de phase (grossissement $x 100$ ) en fonction de la densité cellulaire et entourée par un stylo diamant. Selon la méthode utilisée dans l'équipe et décrite par Bergère et al. [4], nous n'avons pas effectué de décondensation sur les noyaux des cellules testiculaires.

\section{d) Les sondes.}

Les sondes utilisées pour les chromosomes 18 et $X$ sont des sondes centromériques Cep18 (D18Z1) spectre aqua, et CepX (DXZ1) spectre vert, et pour le chromosome $Y$, une sonde de l'hétérochromatine du bras long de I'Y (CEPY DYZ1) spectre orange (Abott). Tous ces sondes ont été conservées à $-20^{\circ} \mathrm{C}$.

\section{e) Hybridation.}

Après dépôt des sondes, les lames ont été disposées sur une plaque chauffante en atmosphère humide (Hybrite, Vysis) à une température de $45^{\circ} \mathrm{C}$ pendant 2 minutes, puis dénaturées à $73^{\circ} \mathrm{C}$ pendant 3 minutes et enfin remise à $37^{\circ} \mathrm{C}$ pour l'hybridation pendant une nuit.

\section{f) Lavage.}

Les lames ont été plongées successivement dans une première solution de lavage (0.4 SSC $0.3 \mathrm{NP} 40$ ) préchauffée dans un bain-marie à $73^{\circ} \mathrm{C} \pm 1^{\circ} \mathrm{C}$ pendant 1 minute et 45 secondes, puis dans une deuxième solution de lavage (2xSSC 0.1NP40) à température ambiante pendant 15 secondes.

\section{g) Contre coloration.}

Les noyaux cellulaires ont été contre colorés au 4,6-diamino 2 phenylindole (DAPI) (15 microlitres), puis chaque lame a été couverte d'une lamelle et laissée à l'obscurité pendant 10 minutes.

\section{h) Lecture.}

La lecture a été effectuée dans une chambre noire le jour même ou après conservation des lames à $+4^{\circ} \mathrm{C}$ à l'obscurité au réfrigérateur. Le contenu chromosomique a été déterminé selon le nombre de signaux détectés pour chaque sonde en utilisant un microscope à fluorescence (Olympus) équipé de différents filtres (rouge, vert, DAPI, turquoise). Les images ont été saisies par une camera $L C D$ reliée à un équipement informatique G4 Macintosh équipé du logiciel d'acquisition des images (Quips Pathvysion Digital Scientific version 1.4) (Figure 1).

\section{Analyse statistique}

La comparaison des résultats concernant le taux d'aneuploïdie dans les deux populations de sujets âgés et de sujets jeunes a été effectuée en utilisant le test de Mann Whitney et le test de khi2 (logiciel StatView).

\section{RESULTATS}

\section{Histologie}

L'étude histomorphométrique a montré que différents para- mètres étaient perturbés par le vieillissement avec une grande variabilité interindividuelle (Figures $2 a, 2 b, 2 c, 2 d$ ). La moyenne des diamètres des tubes diminuait mais l'épaisseur de la membrane propre augmentait chez les hommes âgés. Les échantillons présentant une spermatogenèse complète avaient tous une membrane propre d'épaisseur conservée faible, proche de celle des contrôles. Par contre, dès qu'elle dépassait 10 microns (Figure 3 ) on constatait, à une exception près, la présence soit d'un arrêt de la spermatogenèse, soit d'une fibrose complète.

L'appauvrissement de l'épithélium séminifère était caractérisé par une diminution progressive des cellules de Sertoli, des spermatogonies, des spermatocytes et des spermatozoïdes (Figure 4). Les lignées étaient de plus en plus incomplètes au fur et à mesure que l'âge augmentait. Les variations interindividuelles étaient très importantes ; 17 sujets âgés (environ la moitié) présentaient encore des spermatozoïdes (Figure 4) ; la spermatogenèse peut être maintenue jusqu'à un âge très avancé puisqu'un sujet âgé de 95 ans présentait une spermatogenèse complète. La raréfaction des spermatozoïdes paraissait en relation avec la diminution des spermatogonies et des spermatocytes (Figure 4) et l'arrêt de la spermatogenèse se produisait le plus souvent au stade pachytène.

Concernant les cellules de Leydig, nous avons relevé une augmentation de leur taille avec l'âge (Figure 5).

Cette étude histologique (Figures $2 a, 2 b, 2 c, 2 d$ ) nous a permis de classer les sujets âgés $(n=36)$ en trois groupes : 17 sujets âgés de 61 à 95 ans à spermatogenèse peu dense mais complète (groupe 1 ), 4 sujets à spermiogenèse arrêtée (groupe 2) et 15 sujets (groupe 3 ) avec un arrêt précoce de la spermatogenèse (cellules diploïdes) ou une absence totale de cellules dans les tubes (4 sujets).

\section{FISH}

Dans le groupe $1(n=17)$, seuls 14 sujets présentaient des cellules post méiotiques analysables par FISH en nombre suffisant. Nous avons analysé un total de 4738 cellules post méiotiques chez ces sujets et un total de 4882 cellules post méiotiques chez les sujets jeunes témoins.

Chez les sujets âgés, les cellules normales haploïdes X18 et $Y 18$ représentaient respectivement $50,76 \%$ et $47,95 \%$ contre $50,35 \%$ et $48,55 \%$ chez les témoins et le taux d'aneuploïdie totale étaient identiques chez les sujets âgés et chez le groupe témoin $1,29 \%$ versus $1,10 \%$ (Tableaux 1 et 2). Pour le groupe 2 (sujets avec un arrêt de la spermiogenèse), les pourcentages de cellules X18, Y18 et le taux d'anomalies chromosomiques au niveau des spermatides ( $n=98$ ) étaient respectivement $48 \%, 38 \%$ et $14 \%$. Ce taux de $14 \%$ ( $3 \%$ XY et $11 \%$ pour la disomie 18 ) est significativement plus élevé que les résultats relevés dans les groupes de sujets âgés à spermatogenèse complète et de sujets témoins ; quant au groupe 3 avec un arrêt précoce de la spermatogenèse (cellules diploïdes), nous avons observé $100 \%$ de cellules rondes diploïdes ( $n=100$ par sujet) sans anomalie chromosomique concernant I' $X, Y$ et 18 . Lorsque l'arrêt de la spermatogenèse se situait au stade pachytène les gonosomes n'étaient pas appariés. 


\section{spermatides}

\section{X18 \\ spermatozoïdes}

\section{Y18}
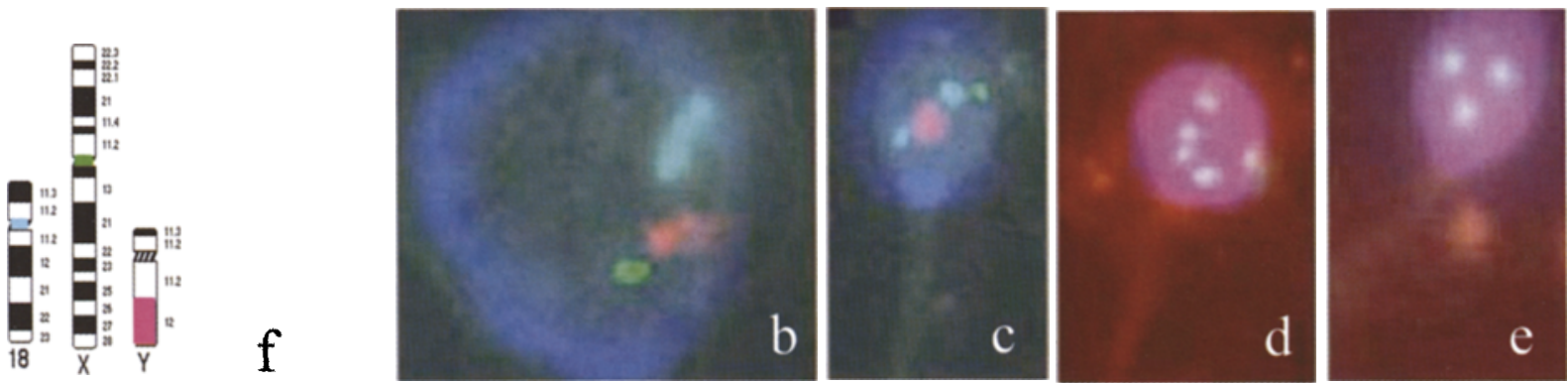

Figure 1 : Exemples de cellules examinées par FISH X,Y et 18. a) En haut de gauche à droite: des spermatides et des spermatozoïdes euplö̈des; b) Spermatocyte l euploïde avec un appariement des gonosomes $X Y$ et des autosomes 18 ; c) Arrêt de la méiose : un spermatozoïde XY1818 ; d) Absence de méiose ll aboutissant à un spermatozoïde XX1818 ; e) Non disjonction de $X$ en méiose II : un spermatozoïde XX18; f) Schéma de la localisation des sondes sur les chromosomes $X$, $Y$ et 18.

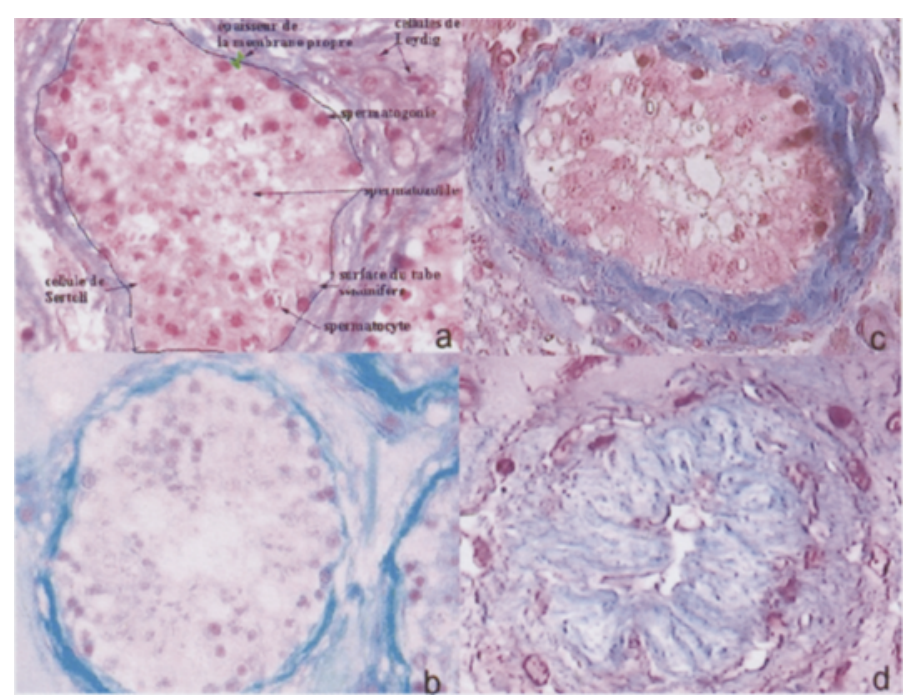

Figure 2 : Histologie testiculaire, a) Coupe histologique au niveau du testicule illustrant les différents paramètres étudiés. b) Spermatogenèse complète à un âge très avancé chez un sujet de 95 ans. c) Epaississement de la membrane propre et arrêt de la spermatogenèse au stade spermatogonie. d) Disparition des lignées cellulaires du parenchyme testiculaire.

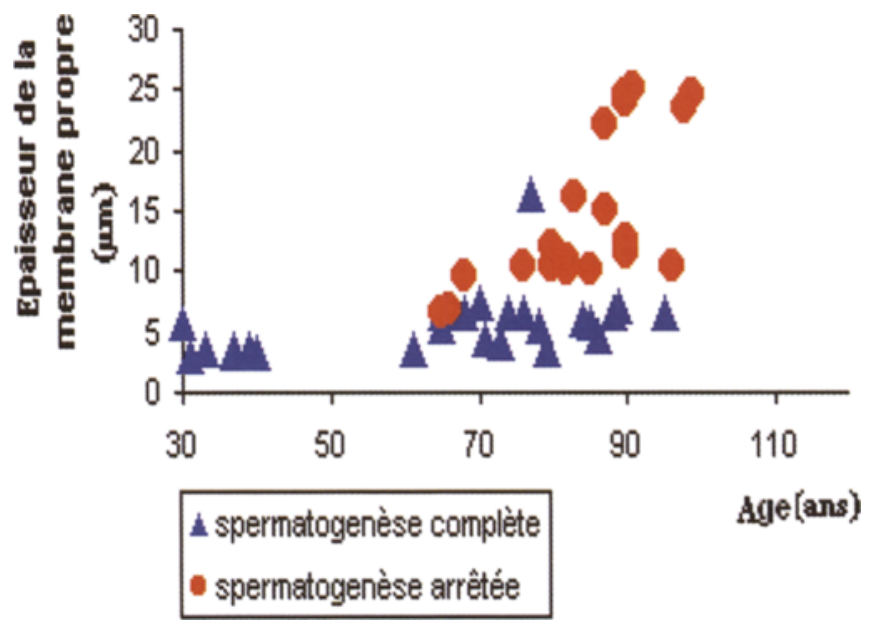

Figure 3 : Epaisseur de la membrane propre en fonction de l'âge et de l'état de la spermatogenèse. 
Nombre de spermatogonies par tube séminifère

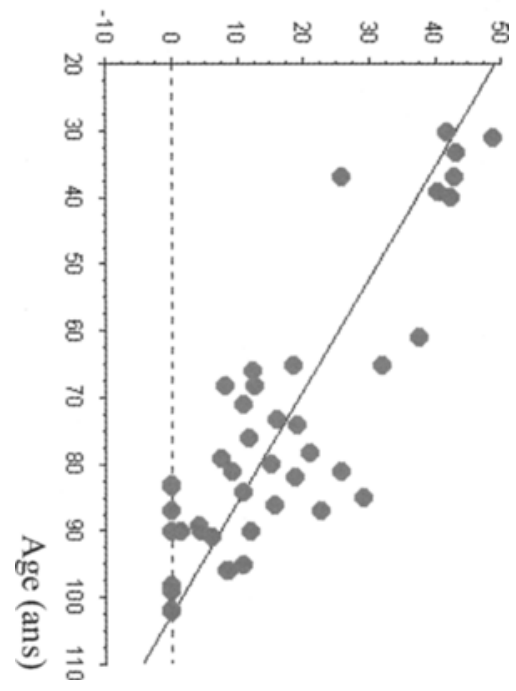

Nombre de cellules de Sertoli par tube séminifère

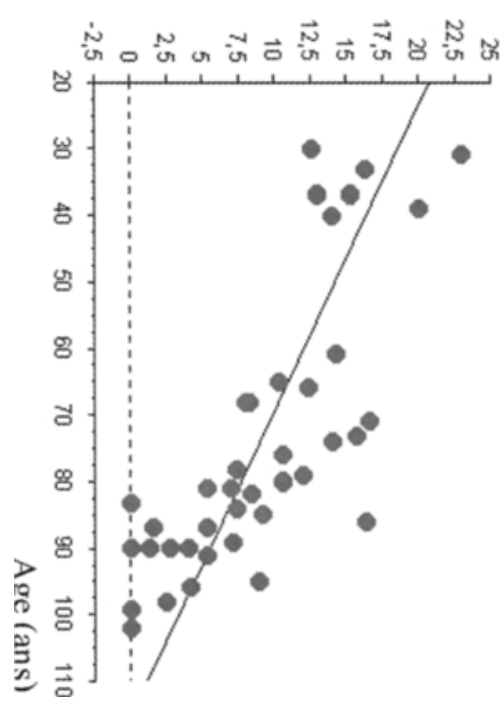

Nombre de spermatocytes par tube séminifère

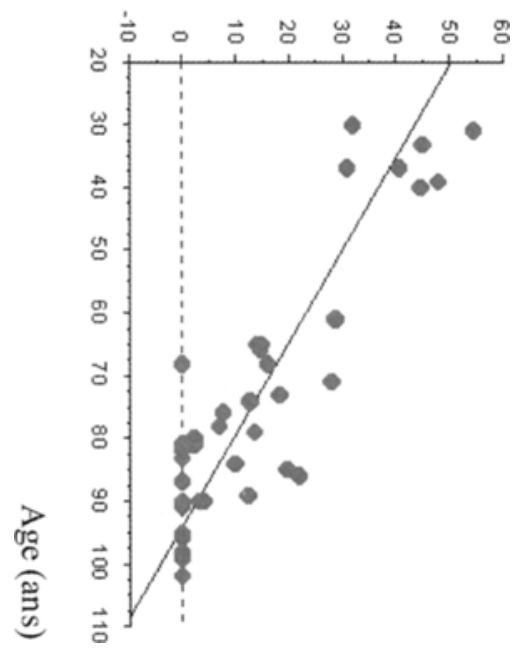

Nombre de spermatozoïdes par tube séminifère

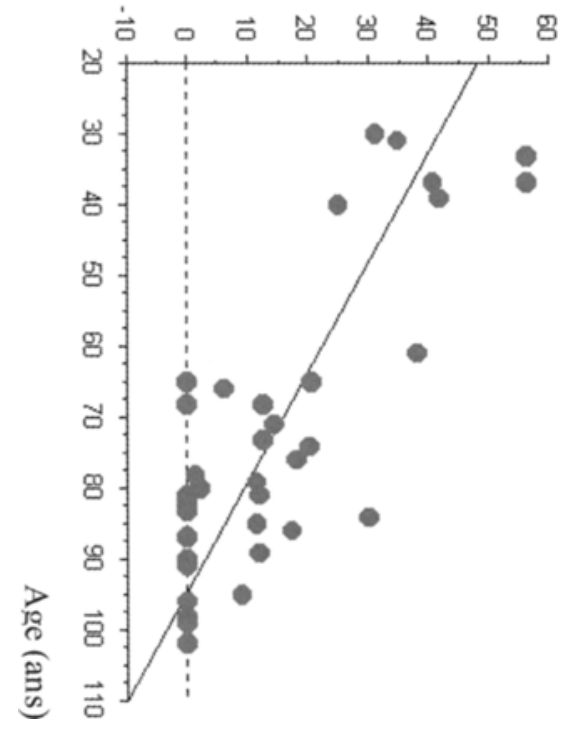

Figure 4 : Evolution du nombre de cellules germinales (les spermatogonies, les spermatocytes et les spermatozoides) et de cellules de Sertoli en fonction de l'âge.

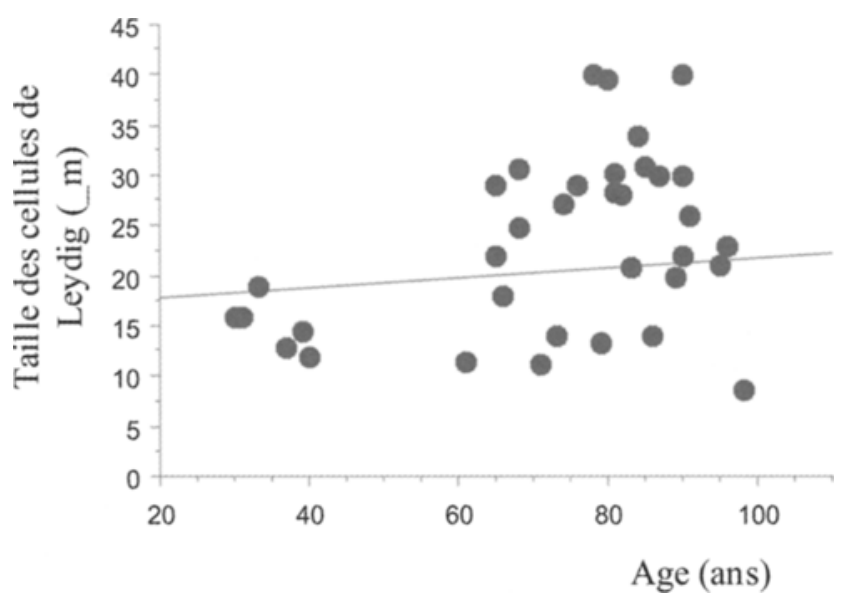

Figure 5 : Evolution de la taille en $\mu m$ des cellules de Leydig en fonction de l'âge. 
Tableau 1 : Incidence des aneuploïdies dans 3 groupes de sujets, témoins, âgés à spermatogenèse préservée, et âgés avec arrêt de la spermiogenèse.

\begin{tabular}{llcc}
\hline & Témoins & $\begin{array}{c}\text { Sujets âgés avec } \\
\text { spermatogenèse préservée }\end{array}$ & $\begin{array}{c}\text { Sujets âgés avec } \\
\text { arrêt de la spermiogenèse }\end{array}$ \\
\hline $\begin{array}{l}\text { Sujets analysés } \\
\text { Nombre de cellules }\end{array}$ & 10 & 14 & 4 \\
post méiotiques & 4882 & 4738 & 98 \\
X18 & $2458(50,34 \%)$ & $2405(50,76 \%)$ & $47(47,96 \%)$ \\
Y18 & $2370(48,6 \%)$ & $2272(47,95 \%)$ & $37(37,75 \%)$ \\
Anomalies chromosomiques & $54(1,10 \%)$ & $61(1,29 \%)$ & $14(14,28 \%)$ \\
Analyse statistique khi2 & & $\mathrm{P}>0,05$ & $\mathrm{P}<0,005$ \\
\hline
\end{tabular}

Tableau 2 : Incidence des anomalies chromosomiques dans les spermatides et les spermatozoïdes chez les témoins et les sujets âgés à spermatogenèse complète.

\begin{tabular}{|c|c|c|c|c|}
\hline & $\begin{array}{l}\text { Type } \\
\text { d'anomalie }\end{array}$ & $\begin{array}{l}\text { Sujets } \\
\text { témoins }\end{array}$ & $\begin{array}{l}\text { Sujets âgés avec } \\
\text { spermatogenèse } \\
\text { complète }\end{array}$ & $\begin{array}{l}\text { Analyse statistique } \\
\text { (Mann Withney) }\end{array}$ \\
\hline Sujets analysés & & 10 & 14 & \\
\hline Nombre de cellules post méiotiques & & 4882 & 4738 & NS \\
\hline nullisomie & $\begin{array}{l}\text { X0 } \\
\text { Y0 } \\
\text { O18 }\end{array}$ & $\begin{array}{l}(20) \mathbf{a} \\
0,40 \% \\
(1) \\
0,02 \% \\
(13) \\
0,27 \%\end{array}$ & $\begin{array}{l}(7) \\
0,15 \% \\
(5) \\
0,11 \% \\
(8) \\
0,17 \%\end{array}$ & $\begin{array}{l}\text { NS } \\
\text { NS } \\
\text { NS }\end{array}$ \\
\hline \multirow{4}{*}{ disomie } & $\begin{array}{l}X Y 18 \\
(M I) b\end{array}$ & $\begin{array}{l}(4) \\
0,08 \%\end{array}$ & $\begin{array}{l}(7) \\
0,15 \%\end{array}$ & NS \\
\hline & $\begin{array}{l}\mathrm{XX18} \\
(\mathrm{M} I \mathrm{II}) \mathrm{c}\end{array}$ & $\begin{array}{l}(2) \\
0,04 \%\end{array}$ & $\begin{array}{l}(5) \\
0,11 \%\end{array}$ & NS \\
\hline & $\begin{array}{l}\text { YY18 } \\
\text { (M II) }\end{array}$ & $\begin{array}{l}(6) \\
0,12 \%\end{array}$ & $\begin{array}{l}(6) \\
0,13 \%\end{array}$ & NS \\
\hline & $\begin{array}{l}\text { X1818 and } \\
\text { Y1818 }\end{array}$ & $\begin{array}{l}(4) \\
0.08 \%\end{array}$ & $\begin{array}{l}(11) \\
0.23 \%\end{array}$ & NS \\
\hline \multirow{3}{*}{ Diploïdie (Arrêt de la méiose) } & $\begin{array}{l}\text { XX1818 } \\
(\mathrm{M} \mathrm{II})\end{array}$ & $\begin{array}{l}(2) \\
0,04 \%\end{array}$ & $\begin{array}{l}(2) \\
0,04 \%\end{array}$ & NS \\
\hline & $\begin{array}{l}\text { YY1818 } \\
\text { (M II) }\end{array}$ & $\begin{array}{l}(1) \\
0,02 \%\end{array}$ & $\begin{array}{l}(1) \\
0,02 \%\end{array}$ & NS \\
\hline & $\begin{array}{l}\text { XY1818 } \\
\text { (MI) }\end{array}$ & $\begin{array}{l}(0) \\
0,0 \%\end{array}$ & $\begin{array}{l}(9) \\
0,19 \% \\
\end{array}$ & NS \\
\hline Autres anomalies chromosomiques & 01818 & $\begin{array}{l}(1) \\
0,02 \%\end{array}$ & $\begin{array}{l}(0) \\
0,00 \%\end{array}$ & NS \\
\hline Effectif total d'aneuploïdies & & $\begin{array}{l}(54) \\
1,1 \%\end{array}$ & $\begin{array}{l}(61) \\
1,29 \%\end{array}$ & NS \\
\hline
\end{tabular}

a : Les chiffres entre parenthèses correspondent au nombre de cellules post méiotiques avec anomalie chromosomique.

$\mathrm{b}: \mathrm{MI}=$ méiose $\mathrm{I} ; \mathrm{c}: \mathrm{MII}=$ méiose $\mathrm{II}$. 
Le détail des différents types d'anomalies retrouvés chez les sujets âgés à spermatogenèse conservée et chez les témoins est donné dans le Tableau 2.

L'analyse de ces résultats avec le test de Mann Whitney ne montre aucune différence statistiquement significative entre les sujets âgés et les témoins.

\section{DISCUSSION}

Dans notre centre, parmi les 1000 consultations annuelles en biologie de reproduction, le nombre de demandes d'assistance médicale à la procréation (AMP) émanant de couples dont le conjoint est âgé de plus 50 ans est d'une vingtaine par an, soit environ $2 \%$, ce qui montre qu'il y a bien un intérêt clinique à évaluer les effets de l'âge sur la spermatogenèse et les accidents génétiques. Pour ce faire, nous avons procédé à la recherche des altérations histologiques et à l'évaluation du risque chromosomique dans la spermatogenèse des sujets âgés de 61 à 102 ans.

Les quelques publications faisant état de résultats chromosomiques $[8,2,21,32,18,31,9,19]$ dans cette situation ne portent que sur un nombre très limité de cas et sur des sujets d'âge beaucoup moins avancé. Ces travaux ont presque tous été effectués sur du sperme éjaculé. Le nombre de sujets âgés dont il est possible d'étudier le sperme éjaculé est donc faible. Au total, si l'on regroupe toutes les études, seulement 14 sujets de plus de 60 ans ont été étudiés de ce point de vue. Nous avons analysé des sujets post mortem, et nous avons travaillé sur du tissu testiculaire. Certes, l'effectif de cellules post méiotiques analysables en FISH au niveau testiculaire est faible en comparaison des spermatozoïdes éjaculés [22], d'autant plus qu'à un âge avancé la production des spermatozoïdes est diminuée (Figure 4). Par contre, nous avons ainsi accès à toutes les étapes de la méiose et à un plus grand nombre de sujets (36 dans notre étude) dont l'âge peut être très avancé (102 ans). De plus, nous pouvons également interpréter nos résultats chromosomiques en fonction de l'histologie testiculaire.

Dans notre série d'hommes âgés de 29 à 102 ans, nous avons relevé une diminution du nombre de cellules de Sertoli avec l'âge et, dans 4 cas, une disparition complète de ces cellules. Ces résultats sont conformes aux travaux antérieurs montrant que les cellules de Sertoli sont les dernières à disparaître [12]. De même, nos résultats montrent l'existence d'une corrélation négative entre le nombre de cellules de la lignée germinale et l'âge, affectant aussi bien les spermatogonies que les spermatocytes et les spermatozoïdes. La diminution de la production de spermatozoïdes peut aussi bien être la conséquence des altérations affectant les cellules de Sertoli [33], que de la disparition des spermatogonies [25]. Cependant dans des situations où le parenchyme testiculaire est altéré, l'arrêt de la spermatogenèse est fréquemment décrit au stade pachytène [12], ce que confirme notre présente étude; dans ce cas en FISH les gonosomes des spermatocytes au stade pachytène n'étaient pas appariés.
Concernant les cellules de Leydig, si certains auteurs rapportent une diminution de leur nombre avec l'âge [13], pour d'autres leur nombre reste stable [14], et enfin certains décrivent une augmentation de leur taille avec l'âge [16]. Compte tenu de la très importante variabilité interindividuelle relevée dans notre étude, nos résultats semblent en faveur de cette dernière observation.

Plusieurs études ont retrouvé une faible augmentation de l'incidence des malségrégations au cours de la méiose chez les hommes âgés de 40 à 60 ans. Ces études ont été effectuées sur du sperme éjaculé. Griffin et al. [8] ont trouvé chez 24 sujets âgés de 18 à 60 ans une corrélation entre l'augmentation de l'âge et la fréquence des disomies $X Y$, $X X$ et $Y Y$ dans les spermatozoïdes. Asada et al. [2] avaient relevé une augmentation de la fréquence des spermatozoïdes $X Y$ chez 10 sujets âgés de plus de 39 ans. Martin et al. [21] ont comparé 7 sujets âgés de 21 à 39 ans et 3 sujets âgés de 44 à 52 ans en notant une augmentation de la fréquence des disomies 1 et YY. Robbins et al. [32] avaient noté une augmentation de l'incidence des disomies $X X$ et $Y Y$ chez 4 sujets âgés de plus de 45 ans (âge moyen 46,8 ans). Lowe et al. [18] ont suggéré un effet de l'âge paternel sur la survenue du syndrome de Klinefelter dans la descendance puisque 4 pères d'enfants Klinefelter les avaient conçus entre 50 et 59 ans alors que la fréquence de leurs spermatozoïdes XY était élevée. Rives et al. [31] ont étudié une série de 12 sujets âgés de plus de 50 ans dont 3 seulement étaient âgés de plus de 60 ans. Dans cette étude une augmentation significative des disomies $X Y, 4,18,21$ ainsi que de la diploïdie a été rapportée.

Par contre d'autre études réalisées chez des sujets plus âgés n'ont pas confirmé ces résultats. Guttenbach et al. [9] ont étudié 3 sujets âgés de 81 ans à 83 ans chez qui ils avaient constaté une faible augmentation de l'incidence des malségrégations pour $X Y$ alors que les autres non-disjonctions n'étaient pas affectées par l'âge. Enfin, l'étude de Luetjens et al. [19] portant sur 8 sujets âgés de plus de 60 ans n'a pas trouvé de différence significative concernant les anomalies méiotiques comparés aux sujets jeunes.

Au total, en reprenant le détail les séries publiées, on retrouve dans la littérature des résultats d'analyse chromosomique des spermatozoïdes pour 14 sujets âgés de plus de 60 ans avec des résultats contradictoires. Dans notre étude de sujets très âgés à spermatogenèse conservée, nous n'avons pas observé d'augmentation de l'incidence des aneuploïdies par rapport aux témoins. Lorsque la spermatogenèse est conservée à des âges très avancés l'augmentation de l'incidence des anomalies chromosomiques avec l'âge, si elle existe, est très faible en valeur absolue. Par contre pour les 4 sujets âgés avec spermiogenèse arrêtée, le taux global d'anomalies chromosomiques était de $14,28 \%$. Ceci suggère une relation entre atteinte de la spermatogenèse et altération méiotique avec l'âge.

En conclusion, le risque de conception d'un enfant porteur d'anomalie chromosomique reste donc faible lorsque le père est âgé. Par contre puisque plusieurs études épidémiologiques ont rapporté une corrélation entre âge paternel 
et risque de mutation autosomique dominante chez la descendance [3], la question qui reste posée est celle du risque de mutation génique au niveau des cellules germinales des sujets âgés.

\section{REFERENCES}

1. ARAN B., BLANCO J., VIDAL F. et al. : Screening for abnormalities of chromosomes $X, Y$, and 18 and for diploidy in spermatozoa from infertile men participating in an in vitro fertilization-intracytoplasmic sperm injection program. Fertil. Steril., 1999, 72 : 696-701.

2. ASADA H., SUEOKA K., HASHIBA T., KUROSHIMA M., KOBAYASHI N., YOSHIMURA Y. : The effects of age and abnormal sperm count on the non disjunction of spermatozoa. J. Assist. Reprod. Genet., 2000, $17: 51-59$.

3. AUROUX M. : Age du père et aptitude au développement. Contracept. Fertil. Sex., 1992, $20: 942-945$.

4. BERGERE M., WAINER R., NATAF V. et al. : Biopsied testis cells of four 47,XXY patients : fluorescence in situ hybridization and ICSI results. Hum. Reprod., 2002, $17: 32-37$.

5. BICCHIERAY L., BENFTIMA I., ALBERT M. et al. : Analyse morphométrique semi quantitative de l'histologie testiculaire au cours du vieillissement. Andrologie, 2003, $13: 288-297$.

6. CROW J.F. : The high spontaneous mutation rate $:$ is it a health risk ? Proc. Natl. Acad. Sci., 1997, $94: 8380-8386$.

7. ESKENAZI B., WYROBEK A.J., SLOTER E. et al. : The association of age and semen quality in healthy men. Hum. Reprod., 2003, $18:$ 447-454.

8. GRIFFIN D.K., ABRUZZO M.A., MILLIE E.A. et al. : Non-disjunction in human sperm : evidence for an effect of increasing paternal age. Hum. Mol. Genet., 1995, 4 : 2227-2232.

9. GUTTENBACH M., KOHN F.M., ENGEL W., SCHMID M. : Meiotic no disjunction of chromosomes $1,17,18, X$, and $Y$ in men more than 80 years of age. Biol. Reprod., 2000, 63 : 17271729.

10.HASSOLD T., BENHAM F., LEPPERT M. : Cytogenetic and molecular analysis of sex-chromosome monosomy. Am. J. Hum. Genet., 1988, 42 : 534-541.

11. HOLSTEIN A.F. : Spermatogenesis in the aged-a borderland between normal and pathologic anatomy. Urologe A, 1986, 25 : 130-137.

12.JOHNSON L.J. : Spermatogenesis and aging in the human. J. Androl., $1986: 7: 331-354$.

13.KALER L.W., NEAVES W.B. : Attrition of the human Leydig cell population with advancing age. Anat. Rec., 1978, $192: 513$ 518.

14.KADEN R. : Testicular histology in the aging man. Fortschr. Med., 1975, 93 : 1545-1549.

15.KIDD S.A., ESKENAZI B., WYROBEK A.J. : Effects of male age on semen quality and fertility. Fertil. Steril., 2001, $75: 237-248$.

16.KOTHARI L.K., GUPTA A.S. : Effect of ageing on the volume, structure and total Leydig cell content of the human testis. Int. J. Fertil., 1974, $19:$ 140-146.

17.LANSAC J. : Delayed parenting. Is delayed childbearing a good thing ? Hum. Reprod., 1995, $10: 1033-1035$.

18.LOWE X., ESKENAZI B., NELSON D.O., KIDD S., ALME A., WYROBEK A.J. : Frequency of $X Y$ sperm increases with age in fathers of boys with Klinefelter syndrome. Am. J. Hum. Genet., 2001, 69: 1046-1054.

19. LUETJENS C.M., ROLF C., GASSNER P., WERNY J.E.,
NIESCHLAG E. : Sperm aneuploidy rates in younger and older men. Hum. Reprod., 2002, 17 : 1826-1832.

20. MANIERI C., FORNENGO R., MOLINATTI G.M. : Male fertility in the elderly. Arch. Ital. Urol. Androl., 1993, 65 : 501-505.

21. MARTIN R.H., SPRIGGS E., KO E., RADEMAKER A.W. : The relationship between paternal age, sex ratios, and aneuploidy frequencies in human sperm, as assessed by multicolor FISH. Am. J. Hum. Genet., 1995, 57 : 1395-1399.

22. MATEIZEL I., VERHEYEN G., VAN ASSCHE E., TOURNAYE H., LIEBAERS I., VAN STEIRTEGHEM A. : FISH analysis of chromosome $\mathrm{X}, \mathrm{Y}$ and 18 abnormalities in testicular sperm from azoospermic patients. Hum. Reprod., 2002, 17 : 22492257.

23. NEAVES W.B., JOHNSON L., PORTER J.C., PARKER C.R. Jr PETTY C.S. : Leydig cell numbers, daily sperm production, and serum gonadotropin levels in aging men. J. Clin. Endocrinol. Metab., 1984, $59:$ 756-763.

24. NICOLAIDIS P., PETERSEN M.B. : Origin and mechanisms of non-disjunction in human autosomal trisomies. Hum. Reprod., 1998, $13:$ 313-319.

25. NISTAL M., CODESAL J., PANIAGUA R., SANTAMARIA L. Decrease in the number of human $A p$ and $A d$ spermatogonia and in the Ap/Ad ratio with advancing age. New data on the spermatogonial stem cell. J. Androl., 1987, $8: 64-68$.

26. PANIAGUA R., AMAT P., NISTAL M., MARTIN A. : Ultrastructural changes in Sertoli cells in ageing humans. J. Androl., 1985, $8: 295-312$.

27. PANIAGUA R., AMAT P., NISTAL M., MARTIN A. Ultrastructure of Leydig cells in human ageing testes. J. Anat., 1986, $146: 173-183$

28. PELLESTOR F., ANDREO B., ARNAL F., HUMEAU C., DEMAILLE J.: Maternal aging and chromosomal abnormalities: new data drawn from in vitro unfertilized human oocytes. Hum. Genet., 2003, 112 : 195-203.

29. PETERSEN P.M., PAKKENBERG B. : Stereological quantitation of Leydig and Sertoli cells in the testis from young and old men. Image Anal. Stereol., 2000, $19: 215-218$.

30. REGADERA J., NISTAL M., PANIAGUA R. : Testis, epididymis, and spermatic cord in elderly men. Correlation of angiographic and histologic studies with systemic arteriosclerosis. Arch. Pathol. Lab. Med., 1985, 109 : 663-667.

31. RIVES N., LANGLOIS G., BORDES A., SIMEON N., MACE B.: Cytogenetic analysis of spermatozoa from males aged between 47 and 71 years. J. Med. Genet., 2002, $39: 63 E$.

32. ROBBINS W.A., BAULCH J.E., MOORE D. 2nd, WEIER H.U., BLAKEY D., WYROBEK A.J. : Three-probe fluorescence in situ hybridization to assess chromosome $X, Y$, and 8 aneuploidy in sperm of 14 men from two healthy groups : evidence for a paternal age effect on sperm aneuploidy. Reprod. Fertil. Dev., 1995, $7: 799-809$

33. RUSSELL L.D., PETERSON R.N. : Determination of the elongate spermatid-Sertoli cell ratio in various mammals. J. Reprod. Fertil., 1984, 70 : 635-641.

34. RYU H.M., LIN W.W., LAMB D.J., CHUANG W., LIPSHULTZ L.I., BISCHOFF F.Z. : Increased chromosome $X, Y$, and 18 nondisjunction in sperm from infertile patients that were identified as normal by strict morphology : implication for intracytoplasmic sperm injection. Fertil. Steril., 2001, $76:$ 879-883.

35. SASANO N., ICHIJO S. : Vascular patterns of the human testis with special reference to its senile changes. Tohoku J. Exp. Med., 1969, 99 : 269-280. 
Bourse 2003 de la SALF

Manuscrit reçu : février 2004 ; accepté : février 2004.

\begin{abstract}
Influence of ageing on spermatogenesis: testicular histomorphometric and cytogenetic study in elderly men (61 to 102 years)

Mbarka DAKOUANE, Lucia BICCHIERAY, Marianne BERGERE, Martine ALBERT, Nathalie LEDEE, François VIALARD, Olivier CUSSENOT, Jacqueline SELVA
\end{abstract}

The harmful effect of maternal age on abnormal meiotic behaviour has been clearly established, but little is known about the effect of paternal age on chromosome malsegregations and the results of studies on this question are fairly controversial. The purpose of this study was to evaluate the influence of ageing on testicular histology and aneuploidy rate in testicular post-meiotic cells. A possible age-related risk has been suggested by the increased frequency of medical assisted reproduction techniques for older men.

We analysed 36 testicular samples from subjects aged 61-102 years by histology and histomorphometry. We examined testicular cells with fluorescence in situ hybridisation (FISH). We studied six histological sections ( 20 crosssectioned tubules) by computer-assisted morphometric analysis (Histolab). The study was based on the following parameters: for seminiferous tubules: surface area, thickness of the basement membrane, nucleus density (Sertoli cells, spermatogonia, spermatocytes and spermatozoids); for interstitial tissue: cluster number and surface area occupied by the Leydig cells, nucleus density. We analysed cells by FISH and a set of three probes $X, Y$ and 18 $\left(A b b o t^{\circ}\right)$. The results were compared to those of a control group of testicular biopsies from 10 subjects (29-40 years) with obstructive azoospermia and normal histology.

Results: The histomorphometric study showed various alterations including: thickening of the basement membrane when spermatogenesis was arrested. The number of germinal cells and the number of Sertoli cells decreased with increasing age and Leydig cell hypertrophy was observed with increasing age. Complete spermatogenesis was observed in men up to the age of 95 years old. The most sensitive step was pachytene. Spermatogonia can persist until the age of 98 years. The 36 elderly men were divided into 3 groups: preserved spermatogenesis (17 subjects; group 1), arrested spermatogenesis (4 subjects; group 2) and early disrupted spermatogenesis with only diploid cells or no cells ( 15 subjects; group 3 ).

For the control group, post-meiotic cells $(n=4,882$ ) showed $50.35 \% \times 18,48.55 \%$ Y18 and $1.1 \%$ of cells with aneuploidy. For elderly subjects with preserved spermatogenesis, post-meiotic cell analysis $(n=4,738)$ showed $50.76 \%$
$\mathrm{X} 18,47.95 \% \mathrm{Y} 18$ and $1.29 \%$ of cells with aneuploidy. Subjects with arrested spermatogenesis presented $47.96 \%$ $\mathrm{X} 18,37.75 \% \mathrm{Y} 18$ and the aneuploidy rate among spermatids $(n=98)$ was $14.28 \%$. This rate was higher than those observed in controls and in group 1 . In conclusion, we observed that spermatogenesis was possible until an advanced age (95 years). There appears to be an increased incidence of post-meiotic aneuploidy in the case of arrested spermatogenesis (14.28 vs $1.10 \%)$. The aneuploidy rate in the group of subjects with preserved spermatogenesis was not statistically different from that observed in the control group.

Key words: ageing men, testis, histomorphometric study, postmeiotic cells, FISH, aneuploidy 